\title{
Paper Synthesis and Failure Correction of Flattop and Cosecant Squared Beam Patterns in Linear Antenna Arrays
}

\author{
Hemant Patidar ${ }^{1}$, Gautam Kumar Mahanti ${ }^{1}$, and Ramalingam Muralidharan ${ }^{2}$ \\ ${ }^{1}$ National Institute of Technology, Durgapur, India \\ ${ }^{2}$ Caledonian College of Engineering, Oman
}

https://doi.org/10.26636/jtit.2017.113417

\begin{abstract}
This paper deals with the synthesis of flattop and cosecant squared beam patterns using the firefly algorithm which is based on metaheuristics. This synthesis is followed by the correction of the radiation patterns when unfortunate malfunctioning of the individual elements in the array occurs. The necessary attention is given to the recovery process, with due emphasis on reduction of side lobe level, ripple and the reflection coefficient. Simulation in Matlab shows a successful employment of the firefly algorithm in producing voltage excitations of the good elements necessary for the recovered patterns. The performance of the firefly algorithm in failure correction is validated by duly comparing it with a standard benchmark.
\end{abstract}

Keywords-cosecant squared pattern, failure correction, firefly algorithm, flattop beam pattern, linear array, side lobe level.

\section{Introduction}

Past decades depict the importance of various shaped beam patterns, like the flattop pattern or the cosecant squared pattern, in a variety of applications relating to telecommunications. Many methods are being developed for the generation of these kinds of patterns and, in addition, importance is also attached to the various parameters that have evolved out of the radiation patterns. The most significant parameter is the side lobe level (SLL) referring to the ratio of the peak of the main lobe to the peak of the side lobe in the antenna array radiation pattern [1]. It is also the most important factor in the optimization of antenna array patterns. The higher SLL level, the bigger deterioration in the pattern that will cause failures in one or more of the antenna elements. These failures are represented by the zero excitations elements. These failures affect not only the SLL, but also many other parameters, like beam width or reflection coefficient. The reflection coefficient (RC) describes how much of an electromagnetic wave is reflected by an impedance discontinuity or a mismatch in the communication medium. The RC value equals the ratio of the amplitude of the reflected wave to the incident wave. Failures disturb the signal and force a correction to the patterns, irrespective of the beams shape.

The best correction method is to replace hardware, if the antenna array is easy to reach. This leads to a further in- crease in cost and difficulty. On the other hand, there are solutions relying on digital beam forming, using evolutionary algorithms, where - by modifying the excitations of good (unfailed) elements - the failures can be corrected in such a way that the recovered radiation pattern resembles that of the original pattern in terms of radiation pattern parameters as well as other parameters.

Literature review reveals many algorithms used in the past that proved to be helpful. Depending on their concepts and characteristics, each is proving to be better than the other.

Rodriguez and Ares [2] recovered the original pattern by changing the amplitude and phase distributions separately for both the sum and shaped beam patterns, successfully using the simulated annealing technique. Yeo and $\mathrm{Lu}$ in [3] presented numerous examples of single and multiple element failure corrections using beam forming weights, representing them directly by a vector of complex numbers. Lozano et al. [4] synthesized the patterns by finding the optimal configuration of the array factor roots, using the simulated annealing technique in both sum and flat-topped beam patterns. Rodriguez et al. applied the genetic algorithm [5] in restoring the array pattern with three failures, by changing the amplitude distribution of less than a third of the unfailed array elements. Yeo and $\mathrm{Lu}$ in [6] utilized particle swarm optimization for the radiation patterns correction during the failure of a few elements in the array.

Active impedance [7] affects the patterns and its corresponding parameters in terms of mutual impedance and self-impedance. Synthesis of antenna arrays with a shade of mutual coupling have been reported in [8]-[10]. To quote a few works in the past concerned with shaped beam patterns, Zhou et al. in [11] realized a 10-element flattop beam shaped linear antenna array, which gave a wellproportioned power distribution in the required zone using the genetic algorithm. Lei et al. [12] ] presented a cosecant squared beam pattern in a wide band integrated linear printed array using a modified least square method by matrix inversion, which can be directly used in surveillance radar applications.

This paper proposes a synthesis of the flat top beam pattern and the cosecant squared beam pattern, using amplitude and 
phase excitations, with a two-bit phase shifter for the flat top beam and continuous phase for the cosecant squared beam.

One of the recent evolutionary firefly algorithms (FA) [13]-[15] has been used here to generate excitations, due to its successful past usage in the field of antenna arrays [15]. The novelty of the approach presented in this paper consists in the fact that the failure correction process is performed for different shaped beam patterns, and active impedance is duly taken into consideration during the processing of both far-field patterns. The proposed algorithm is compared with the well-known Particle Swarm Optimization (PSO) algorithm [16]-[17].

\section{Problem Formulation}

The far field pattern in $\mathrm{x}-\mathrm{y}$ plane for the linear antenna array constructed of parallel half wavelength $(\lambda)$ dipoles equally spaced at a distance $d=0.48 \lambda$ is given by:

$$
F F P(\phi)=\left[\sum_{n=1}^{N} C_{n} \mathrm{e}^{j(n-1) k d \cos \phi}\right] \cdot \text { Element } P(\phi),
$$

where $n$ represents the element number, $N$ is the total number of array elements, $\phi$ is the angle measured from the $\mathrm{x}$ axis to the far-field point, $k$ is the wave number and $C_{n}$ is the excitation current of $n$-th element.

The element pattern of each dipole Element $P$ is assumed to be omnidirectional within the plane taken into consideration, and is substituted by a value equal to unity. The expression for the self and mutual impedances are taken from [7]. A sum pattern is produced in the broadside direction using the current excitations obtained with the help of the mutual coupling impedance matrix.

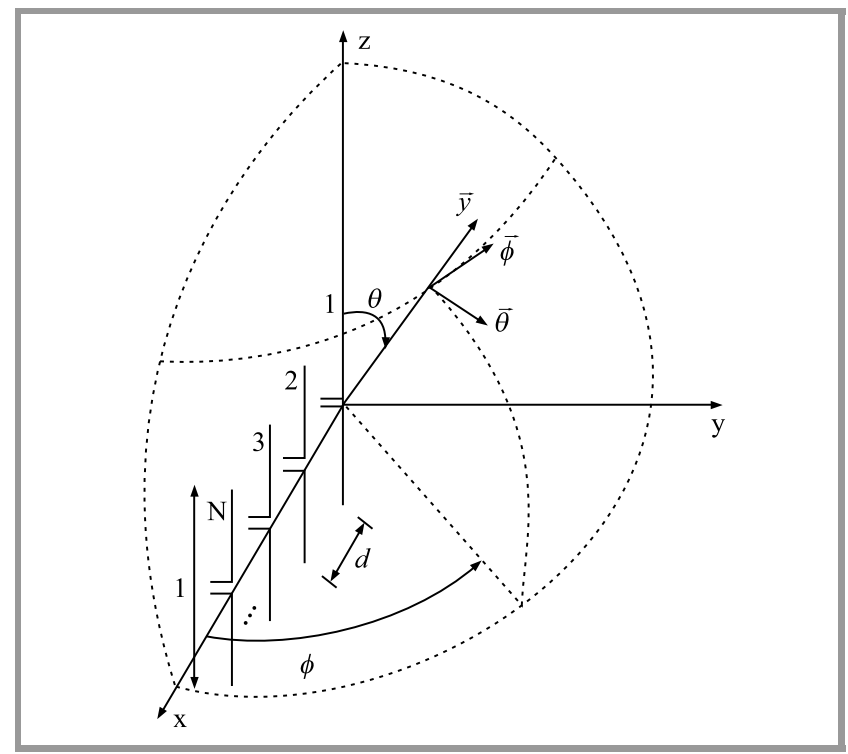

Fig. 1. Geometry of a linear antenna array with elements in the $\mathrm{x}$ axis and placed parallel to $\mathrm{z}$ axis.
Considering the characteristic impedance $Z_{0}$ of the feed network to be $50 \Omega$, the reflection coefficient RC (in $\mathrm{dB}$ ) at the input of the $a$-th dipole antenna is given by:

$$
\mathrm{RC}=20 \log \frac{\left|Z_{a}^{A}\right|-Z_{0}}{\left|Z_{a}^{A}\right|+z_{0}},
$$

where $Z_{a}^{A}$ is the active impedance of the elements. From among all the elements in the array, the maximum of the $\mathrm{RC}$ value is obtained. The active impedance of any failed element is considered to be zero. The problem is to obtain a new set of voltage excitations of the remaining non-failed elements such that the corrected pattern resembles similar to that of the original pattern in terms of the expected radiation pattern parameters, i.e. SLL, ripple in the flat top portion of the pattern, and RC. Similarly, for the restoration of the cosecant squared pattern, a new set of voltage and phase excitations for the remaining good elements is generated in such a way that the corrected restored pattern using the newly generated excitations resembles the original pattern in terms of radiation pattern parameters.

The fitness function for generation of the original and the corrected flattop beam pattern is:

$$
\text { Fitness }=w t_{1} F_{1}^{2}+w t_{2} F_{2}^{2}+w t_{3} F_{3}^{2},
$$

where:

$$
\begin{gathered}
F_{1}= \begin{cases}\mathrm{SLL}_{\mathrm{o}}-\mathrm{SLL}_{\mathrm{d}} & \text { if } \mathrm{SLL}_{\mathrm{o}}>\mathrm{SLL}_{\mathrm{d}} \\
0 & \text { if } \mathrm{SLL}_{\mathrm{o}} \leq \mathrm{SLL}_{\mathrm{d}}\end{cases} \\
F_{2}=\left\{\begin{array}{ll}
\mid \text { ripple }_{\max }^{\mathrm{o}} \mid- \text { ripple }_{\max }^{\mathrm{d}} & \text { if } \mid \text { ripple }_{\max }^{\mathrm{o}} \mid>\text { ripple }_{\max }^{\mathrm{d}} \\
0 & \text { if } \mid \text { ripple }_{\max }^{\mathrm{o}} \mid \leq \text { ripple }_{\max }^{\mathrm{d}}
\end{array},\right. \\
F_{3}= \begin{cases}\mathrm{RC}_{\max }^{\mathrm{o}} & \text { if } \mathrm{RC}_{\max }^{\mathrm{o}}>\mathrm{RC}_{\max }^{\mathrm{d}} \\
0 & \text { if } \mathrm{RC}_{\max }^{\mathrm{o}} \leq R C_{\max }^{\mathrm{d}}\end{cases}
\end{gathered}
$$

In Eq. (3), ripple refers to the difference between the maximum and the minimum value of the far-field pattern in the desired flat top sector pattern. The "o" prefix stands for the obtained values and "d" refers to the desired values with the "max" suffix referring to the maximum obtained value. The fitness function for generation of the original and the corrected cosecant squared pattern is:

$$
\text { Fitness }=w t_{1} F_{1}^{2}+w t_{2} F_{2}^{2}+w t_{3} F_{3}^{2}+w t_{4} F_{4}^{2},
$$

where:

$$
\begin{gathered}
F_{1}=\left\{\begin{array}{ll}
\mathrm{SLL}_{\mathrm{o}}-\mathrm{SLL}_{\mathrm{d}} & \text { if } \mathrm{SLL}_{\mathrm{o}}>\mathrm{SLL}_{\mathrm{d}} \\
0 & \text { if } \mathrm{SLL}_{\mathrm{o}} \leq \mathrm{SLL}_{\mathrm{d}}
\end{array},\right. \\
F_{2}=\left\{\begin{array}{ll}
\left|\mathrm{rpl}_{\max }^{\mathrm{o}}\right|-\mathrm{rpl}_{\max }^{\mathrm{d}} & \text { if }\left|\mathrm{rpl}_{\max }^{\mathrm{o}}\right|>\mathrm{rpl}_{\max }^{\mathrm{d}} \\
0 & \text { if }\left|\mathrm{rpl}_{\max }^{\mathrm{o}}\right| \leq \mathrm{rpl}_{\max }^{\mathrm{d}}
\end{array},\right. \\
F_{3}=\left\{\begin{array}{ll}
\mathrm{RC}_{\max }^{\mathrm{o}}-\mathrm{RC}_{\max }^{\mathrm{d}} & \text { if } \mathrm{RC}_{\max }^{\mathrm{o}}>\mathrm{RC}_{\max }^{\mathrm{d}} \\
0 & \text { if } \mathrm{RC}_{\max }^{\mathrm{o}} \leq \mathrm{RC}_{\max }^{\mathrm{d}}
\end{array},\right. \\
F_{4}=\left\{\begin{array}{lll}
\left|\mathrm{rpc}_{\max }^{\mathrm{o}}\right|-\mathrm{rpc}_{\max }^{\mathrm{d}} & \text { if }\left|\mathrm{rpc}_{\max }^{\mathrm{o}}\right|>\mathrm{rpc}_{\max }^{\mathrm{d}} \\
0 & \text { if }\left|\mathrm{rpc}_{\max }^{\mathrm{o}}\right| \leq \mathrm{rpc}_{\max }^{\mathrm{d}}
\end{array}\right.
\end{gathered}
$$


In Eq. (4), rpl and rpc refers to the difference between the maximum and the minimum value of the far-field pattern in the desired flat portion $\left(90\right.$ to $\left.100^{\circ}\right)$ and the cosecant squared beam portion $\left(100\right.$ to $\left.145^{\circ}\right)$ in the pattern.

\section{Firefly Algorithm}

The firefly algorithm [13]-[14] is based on the social behavior of fireflies or lightning bugs living in tropical and moderate regions and known for their conspicuous usage of bioluminescence during twilight to attract their corresponding mates or prey. Only three rules related with fireflies are used here. All the fireflies are attracted to each other irrespective of their gender. The degree of attractiveness is proportional to their brightness. The darker ones will move towards the brighter ones. However, the apparent brightness decreases as their mutual distance increases. If no fireflies are available whose brightness would be higher than a given value, the other fireflies will start moving randomly.

The steps involved in this algorithm are given as below.

Initialization. The algorithm starts with the initialization of the location of $M$ fireflies in $D$ dimensional search space, with the searching boundary given by:

$$
x_{m d}(0)=\operatorname{rand}_{m d}(0,1)\left(x_{m d}^{\text {upper }}-x_{m d}^{\text {lower }}\right)+x_{m d}^{\text {lower }},
$$

where $m=1,2, \ldots, M$ and $d=1,2, \ldots, D$. The $x_{m d}^{\text {upper }}$ and $x_{m d}^{\text {lower }}$ are the corresponding upper limit and lower limits of the $d$-th variable in the overall population with rand being the uniformly distributed random value.

Light intensity. The next step is to determine the light intensity (brightness) of each firefly at the current iteration using the fitness function for optimization. This intensity is inversely proportionate to the fitness function for every firefly.

Updating of the locations. Each firefly moves towards a brighter firefly and updates its position for the next iteration. The attractiveness factor $\beta$ is relative and is also judged by other fireflies. In other words, it will vary with the distance between two fireflies. In addition, light intensity decreases with the distance from its source, and light is also absorbed by the medium. Thus, it is obvious to say that the light intensity varies according to the inverse square law. For a given medium with a constant light absorption coefficient, the light intensity $I$ varies with the distance $r$, $I=I_{0} \mathrm{e}^{-\gamma r}$, where $I_{0}$ is the original light intensity level. To use the combined influence of absorption as well as the inverse square law, the light intensity is approximated using the Gaussian form as $I=I_{0} \mathrm{e}^{-\gamma r^{2}}$.

As the attractiveness of a firefly is proportionate to the light intensity observed by other fireflies, the firefly attractiveness $\beta$ is $\beta=\beta_{0} \mathrm{e}^{-\gamma r^{2}}$, where $\beta_{0}$ is the attractiveness at $r=0$. The attractiveness between the fireflies is:

$$
x_{m}=x_{m}+\beta_{0} \mathrm{e}^{-\gamma r_{m p}^{2}}\left(x_{m}-x_{p}\right)+\alpha \varepsilon_{m},
$$

where $\gamma$ is the light absorption coefficient whose value depends on the characteristic features of the medium but is fixed in a given medium, $\alpha$ is the randomization parameter varying between 0 and $1, \varepsilon_{m}$ is a vector of random numbers drawn from an uniform of Gaussian distribution, and the attractiveness between the two fireflies $m$ and $p$ is given by the product of $\beta_{0}$ and $\mathrm{e}^{-\gamma r_{m p}^{2}}$ terms.

The Cartesian distance between two $m$ and $p$ fireflies is given at $x_{m}$ and $x_{p}$ by:

$$
r_{m p}=\left\|x_{m}-x_{p}\right\|=\sqrt{\sum_{d=1}^{D}\left(x_{m, d}-x_{p, d}\right)^{2}} .
$$

The algorithm states that the brightest firefly cannot move in any direction, whereas the remaining fireflies modify their location accordingly at current generation. This process is used to obtain the overall best result at the end of the final specified number of iterations. The brightness of the brightest firefly is regarded as the final best fitness value.

The firefly algorithm parameters are as follows: randomness $=1$, minimum value of $\beta=0.2$ and absorption coefficient $=1$. The settings for PSO are: inertial weight $=$ time-varying inertia weight changing randomly between $U$ $(0.4,0.9)$ with iterations, time-varying acceleration coefficients $c_{1}(\mathrm{t})$ and $c_{2}(\mathrm{t})$ to be 1.495 over the full range of the search.

\section{Simulated Results}

\subsection{Flat Top Beam Pattern}

A linear antenna array containing 24 elements with spacing of $0.48 \lambda$ along the $\mathrm{x}$ axis and situated parallel to $\mathrm{z}$ axis is taken into account for simulation purposes. The voltage excitation ranges from 0 to 1 with the binary phase shifter generating only 0 and $180^{\circ}$ phases. Moreover, the choice of the initial population is random and the population size is 48. The original pattern is obtained using FA and is scheduled for 10 runs with 1000 iterations. For the generation of the original pattern, the values of $w t_{1}, w t_{2}$ and $w t_{3}$ are kept at 5, 15 and 1 respectively. The corrected pattern is run 20 times with 1500 iterations each. And for the corrected pattern, the values they are kept at 1, 3 and 3 . The choice of these values denotes the importance of the concerned designed parameter associated therewith. Seven elements out of $24(29 \%)$ are taken as failed: $\mathrm{V}(3)=0$, $\mathrm{V}(6)=0, \mathrm{~V}(11)=0, \mathrm{~V}(14)=0, \mathrm{~V}(17)=0, \mathrm{~V}(20)=0$, and $\mathrm{V}(22)=0$. The choice of defective elements is random.

Table 1 shows that the original pattern without any failures is generated by using the firefly algorithm as per the desired ones. A random choice of 7 element failures deteriorates the pattern resulting in a very poor SLL, as well as ripples and RC. The two algorithms are made to engage in generating the necessary amplitude and the binary phases of the remaining unfailed elements and the results obtained through the algorithms shows that the FA has flared far 
Table 1

Desired and obtained values of parameters for the flattop beam pattern

\begin{tabular}{|l|c|c|c|c|c|}
\hline \multirow{2}{*}{ Parameters } & \multirow{2}{*}{ Desired values } & \multicolumn{4}{c|}{ Patterns } \\
\cline { 3 - 6 } & & \multirow{2}{*}{ Original FA } & Damaged & $\begin{array}{c}\text { Corrected } \\
\text { using FA }\end{array}$ & $\begin{array}{c}\text { Corrected } \\
\text { using PSO }\end{array}$ \\
\hline \hline SLL [dB] & -25 & -25.278 & -5.5891 & -24.9873 & -23.8248 \\
\hline Maximum ripple $\left(70\right.$ to $110^{\circ}$ ) [dB] & \pm 0.5 & 0.40283 & 4.3745 & 0.54535 & 1.529 \\
\hline Maximum reflection coefficient & 0.25 & 0.24786 & 4.3745 & 0.25793 & 0.53846 \\
\hline Mean & - & - & - & 32.12 & 175.83 \\
\hline Standard deviation & - & - & - & 11.82 & 62.72 \\
\hline Computation time [s] & - & 5661 & - & 16854 & 17032 \\
\hline
\end{tabular}

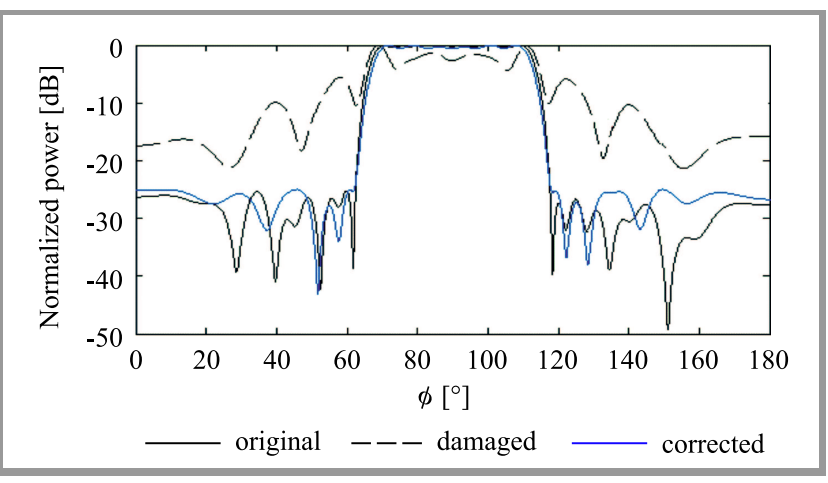

Fig. 2. Original, damaged and corrected normalized power pattern versus $\phi$ for flattop pattern using FA.

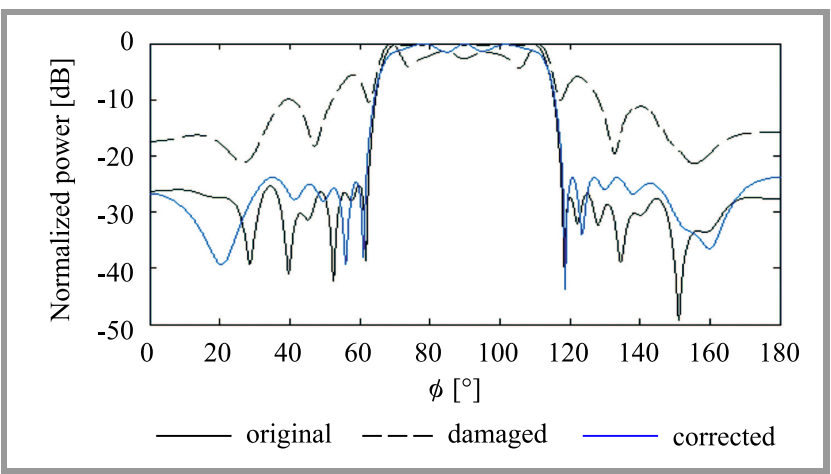

Fig. 3. Original, damaged and corrected normalized power pattern versus $\phi$ for flattop pattern using PSO.

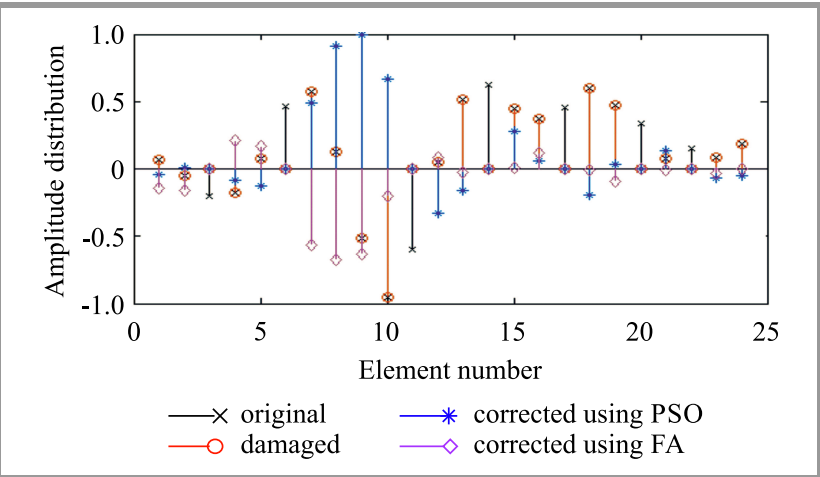

Fig. 4. Amplitude excitations vs. number of elements.

better than the PSO algorithm. Even though FA could not fully meet the needs, it resulted in producing parameters that are very close to the desired values. A magnitude deficit of $0.0127 \mathrm{~dB}$ in SLL, $0.0454 \mathrm{~dB}$ in maximum ripple and 0.00793 in RC is definitely a very low value and this shows a good agreement between the desired and corrected values obtained by using FA. A comparison with PSO also reveals that FA has flared far better than PSO in terms of all parameter values. This is sufficient enough to prove that the former algorithm is better than PSO. The patterns are shown in graphical form in Figs. 2 and 3. Figure 4 shows the corresponding amplitude distributions. A negative value in amplitude distribution represents a $180^{\circ}$ phase.

Table 2

Desired and obtained values of parameters for the cosecant squared beam pattern

\begin{tabular}{|l|c|c|c|c|c|}
\hline \multirow{2}{*}{ Parameters } & \multirow{2}{*}{ Desired values } & \multicolumn{4}{c|}{ Patterns } \\
\cline { 3 - 6 } & & \multirow{2}{*}{ Original FA } & \multirow{2}{*}{ Damaged } & $\begin{array}{c}\text { Corrected } \\
\text { using FA }\end{array}$ & $\begin{array}{c}\text { Corrected } \\
\text { using PSO }\end{array}$ \\
\hline \hline SLL [dB] & -20 & -21.2463 & -6.3317 & -19.691 & -19.3237 \\
\hline Maximum ripple $\left(90\right.$ to $\left.100^{\circ}\right)[\mathrm{dB}]$ & \pm 0.5 & 0.2024 & 3.0963 & 0.53363 & 1.6616 \\
\hline Maximum reflection coefficient & 0.25 & 0.25698 & 0.34199 & 0.27632 & 0.36964 \\
\hline Maximum ripple $\left(100\right.$ to $\left.145^{\circ}\right)[\mathrm{dB}]$ & 1 & 0.99394 & 5.1404 & 1.3294 & 1.8336 \\
\hline Mean & - & - & - & 81.64 & 129.42 \\
\hline Standard deviation & - & - & - & 68.47 & 79.83 \\
\hline Computation time $[\mathrm{s}]$ & - & 4200 & - & 25578 & 26428 \\
\hline
\end{tabular}




\subsection{Cosecant Squared Beam Pattern}

A linear antenna array of 18 elements along the $\mathrm{x}$ axis and situated parallel to the $\mathrm{z}$ axis is taken into account for simulation purposes. The element spacing is $0.48 \lambda$. The original pattern is run 10 times with 1000 iterations each and the population size is 36 . The corrected pattern is run 20 times with 1500 iterations each and the population size is 72 . For the generation of both the patterns using FA, the values of $w t_{1}, w t_{2}, w t_{3}$ and $w t_{4}$ are kept uniform. Five elements out of 18 are taken as failed: $\mathrm{V}(3)=0, \mathrm{~V}(5)=0$,

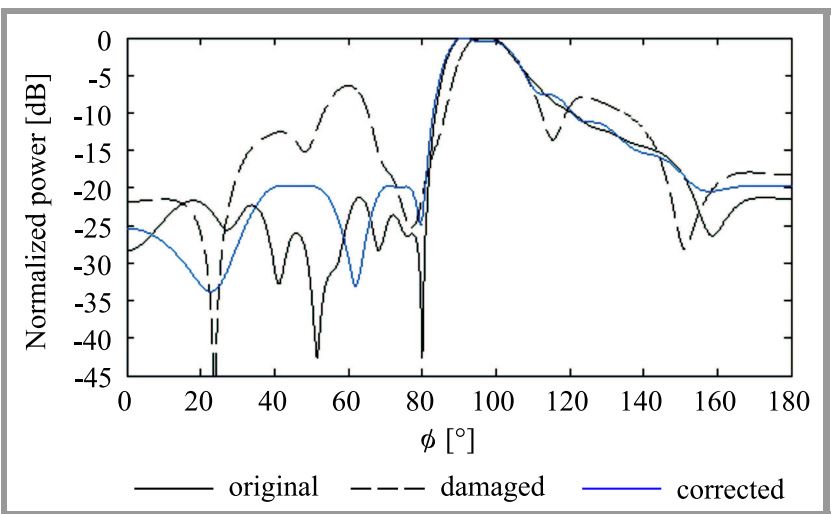

Fig. 5. Original, damaged and corrected normalized power pattern versus $\phi$ for cosecant squared pattern using FA.

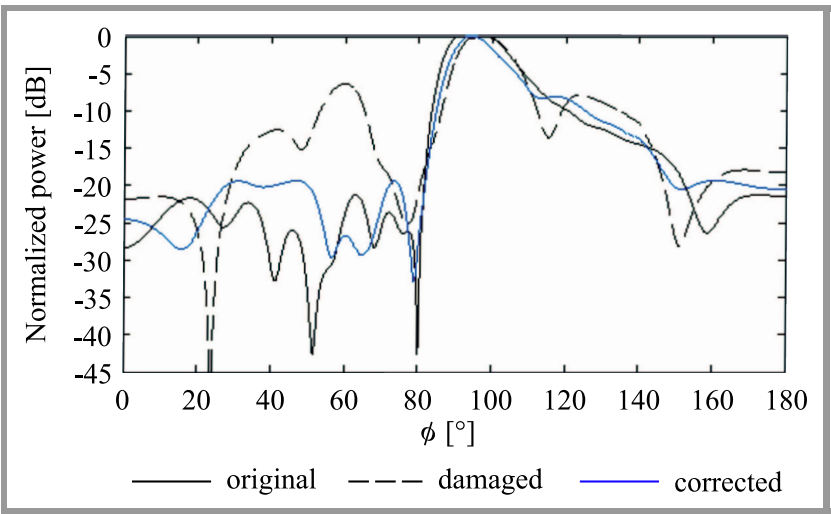

Fig. 6. Original, damaged and corrected normalized power pattern versus $\phi$ for cosecant squared pattern using PSO.

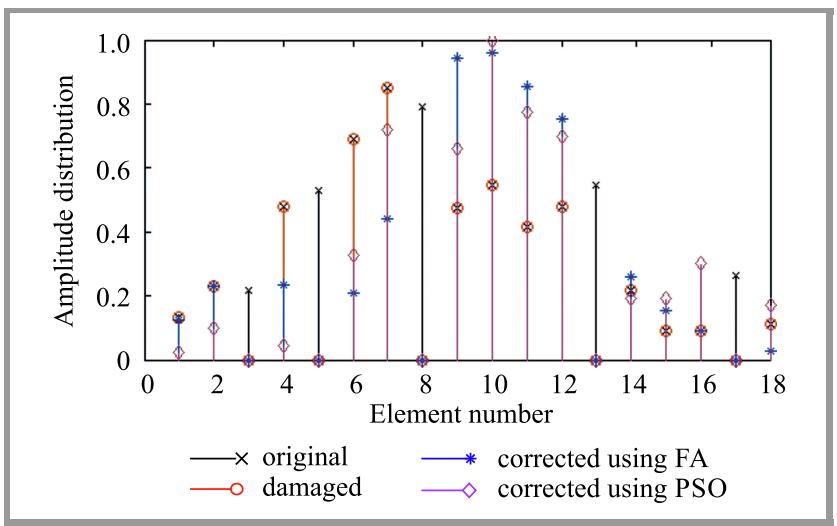

Fig. 7. Amplitude distribution vs. number of elements.
$\mathrm{V}(8)=0, \mathrm{~V}(13)=0, \mathrm{~V}(17)=0$. The choice of the defective element is random. The voltage excitation ranges from 0 to 1 and phase ranges from -180 to $180^{\circ}$.

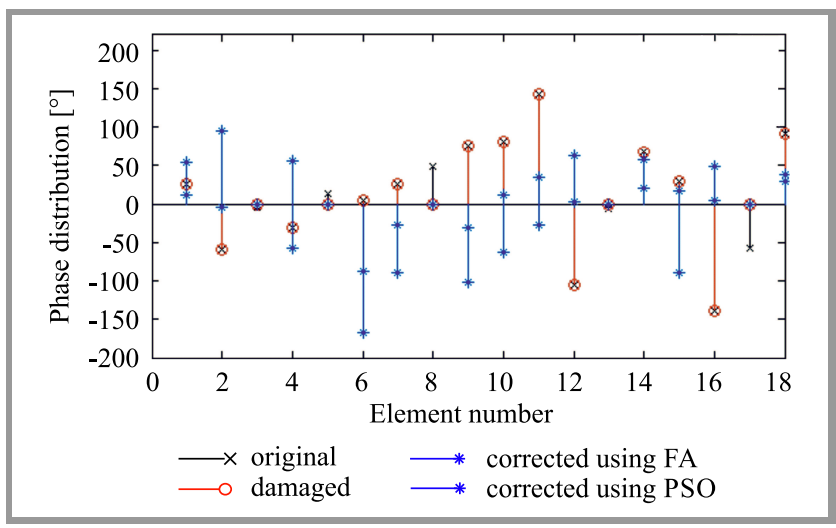

Fig. 8. Phase distribution vs. number of elements.

The original pattern is successfully generated by the FA algorithm. Both algorithms are made to engage in the recovery process and Table 2 shows that FA has once again flared better than PSO. A magnitude deficit of $0.309 \mathrm{~dB}$ in SLL, $0.03363 \mathrm{~dB}$ in ripple (flat), 0.02632 in RC and $0.3294 \mathrm{~dB}$ in ripple (shaped beam) is quite an acceptable one and this also shows that there is a good agreement with the desired and corrected values in the event of a failure. Table 2 also shows that FA has flared far better than PSO in the recovery process. The patterns are shown in Figs. 5 and 6. Figures 7 and 8 show amplitude and phase distributions.

\section{Conclusion}

The simulation performed with the use of Matlab proves successful usage of FA in most of the cases, and it edges better over the benchmark algorithm in restoring the radiation pattern and its associated antenna parameters. This work can be extended in the future to failure correction of shaped beam patterns in other geometries of antenna arrays.

\section{References}

[1] C. A. Balanis, Antenna Theory: Analysis and Design, 2nd ed. Singapore: Wiley (Asia), 2003.

[2] J. A. Rodriguez and F. Ares, "Optimization of the performance of arrays with failed elements using the simulated annealing technique", J. of Electromag. Waves and Appl., vol. 12, no. 12, pp. 1625-1637, 1998.

[3] B. K. Yeo and Y. Lu, "Array failure correction with a genetic algorithm", IEEE Trans. on Antenn. and Propagat., vol. 47, no. 5, pp. 823-828, 1999.

[4] M. V. Lozano, J. A. Rodríguez, and F. Ares, "Recalculating linear array antennas to compensate for failed elements while maintaining fixed nulls", J. of Electromag. Waves and Appl., vol. 13, no. 3, pp. 397-412, 1999.

[5] J. A. Rodriquez, F. Ares, E. Moreno, and G. Franceschetti, "Genetic algorithm procedure for linear array failure correction", Electron. Lett., vol. 36, no. 3, pp. 196-198, 2000. 
[6] B. K. Yeo and Y. Lu, "Fast array failure correction using improved particle swarm Optimization", in Proc. Asia Pacific Microw. Conf. APMC 2009, Singapore, 2009, pp. 1537-1540.

[7] R. S. Elliott, Antenna Theory and Design. New York, USA: Prentice Hall, 1981.

[8] M. Thevenot et al., "Synthesis of antenna arrays and parasitic antenna arrays with mutual couplings", Int. J. of Antenn. and Propagat., vol. 2012, pp. 1-22, 2012.

[9] J. A. Rodriguez, F. Ares, E. Moreno, and G. Franceschetti, "Design of efficient, easily feed matched array antennas by joint optimization of excitations and element geometry: a pencil beam example", Electron. Lett., vol. 34, no. 13, pp. 1280-1282, 1998.

[10] J. A. Rodriguez, F. Ares, and E. Moreno, "Feeding in-phase dipole arrays: a tutorial and a MATLAB program", IEEE Antenn. and Propagat. Mag., vol. 47, no. 5, pp. 169-173, 2005.

[11] H. J. Zhou, Y. H. Huang, B. H. Sun, and Q. Z. Liu, "Design and realization of a flat-top shaped-beam antenna array", Progress in Electromag. Res. Lett., vol. 5, pp. 159-166, 2008.

[12] J. Lei, G. Fu, L. Yang, and D.-M. Fu, "Wide band linear printed antenna array with low sidelobe cosecant square-shaped beam pattern", Progress in Electromag. Res. C, vol. 15, pp. 233-241, 2010.

[13] X. S. Yang, "Firefly algorithm, stochastic test functions and design optimization", Int. J. of Bio-Inspired Comput., vol. 2, no. 2, pp. 78-84, 2010.

[14] X. S. Yang, "Firefly Algorithm, Lévy Flights and Global Optimization", in Research and Development in Intelligent Systems XXVI, M. Bramer, R. Ellis, and M. Petridis, Eds. London: Springer 2010, pp. 209-218.

[15] R. Muralidharan, A. Vallavaraj, and G. K. Mahanti, "Firefly Algorithm for failure correction of linear array of dipole antennas in presence of ground plane with mutual coupling effects", Applied Computat. Electromag. Soc. J., vol. 30, no. 10, pp. 1122-1128, 2015.

[16] J. Kennedy and R. Eberhart, "Particle swarm optimization", in Proc. of the IEEE Int. Conf. on Neural Networks, Perth, Australia, 1995, pp. 1942-1948.

[17] D. W. Boeringer and D. H. Werner, "Particle swarm optimization versus genetic algorithms for phased array synthesis", IEEE Trans. Antenn. Propagat., vol. 52, no. 3, pp. 771-779, 2004.

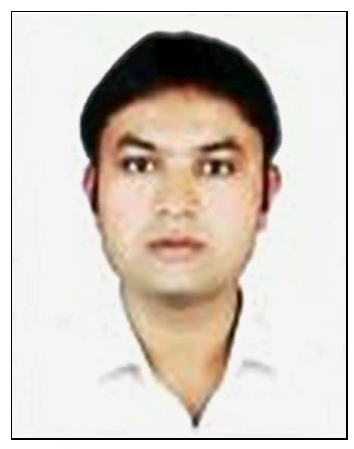

Hemant Patidar obtained his B.Eng. degree in Electronics \& Communication Engineering from Swami Vivekananda College of Engineering, Indore, India in 2012. Since 2013 he has been working as a Ph.D. research scholar in the department of ECE, National Institute of Technology, Durgapur, India. His research interests include array antenna synthesis, evolutionary algorithms, electromagnetics.
E-mail: hemantpatidar08@gmail.com

Department of Electronics and Communication

Engineering

National Institute of Technology

Durgapur, India

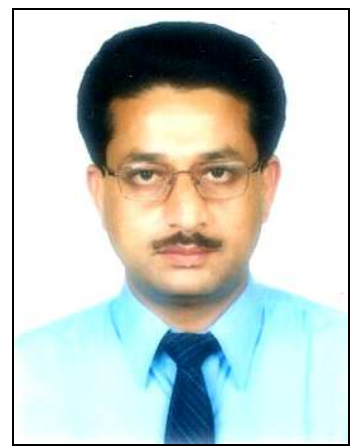

Gautam Kumar Mahanti obtained his B.Eng. in Electronics and Communication Engineering in 1988 from NIT, Durgapur, India, M.Sc. in Electronics Systems and Communication in 1994 from NIT, Rourkela, India, and Ph.D. (Eng.) from IIT, Kharagpur, India. He has more than 20 years of teaching and research experience. Presently he is working as a Professor at the Department of Electronics and Communication Engineering, National Institute of Technology, Durgapur, India. He is a senior member of IEEE, USA. His research area includes array antenna synthesis, evolutionary algorithms and electromagnetics.

E-mail: gautammahanti@yahoo.com

Department of Electronics and Communication

Engineering

National Institute of Technology

Durgapur, India

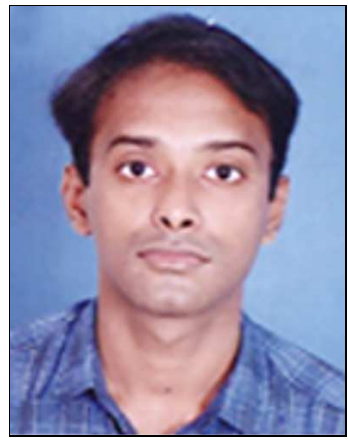

Ramalingam Muralidharan obtained his B.Eng. in Electronics and Communication Engineering in 1995 and M.Sc. (Eng.) in Applied Electronics in 2004, and Ph.D. in 2014 in the field of antenna arrays. He has more than 15 years of teaching experience in teaching subjects related to electronics and communication engineering. Presently, he is working as a Senior Lecturer, at the Department of Electrical and Computer Engineering, Caledonian College of Engineering, Oman. His research areas include array antenna failure correction and evolutionary algorithms.

E-mail: muralidharan@caledonian.edu.om Department of Electrical and Computer Engineering Caledonian College of Engineering

Sultanate of Oman 